\title{
Larger and more heterogeneous neutron star crusts: A result of strong magnetic fields
}

\author{
Jianjun Fang, ${ }^{1, *}$ Helena Pais, ${ }^{1, \dagger}$ Sidney Avancini, ${ }^{1,2, \ddagger}$ and Constança Providência ${ }^{1, \S}$ \\ ${ }^{1}$ CFisUC, Department of Physics, University of Coimbra, 3004-516 Coimbra, Portugal \\ ${ }^{2}$ Departamento de Física, Universidade Federal de Santa Catarina, Florianópolis, SC, CP. 476, CEP 88.040-900, Brazil
}

(Received 13 January 2016; revised manuscript received 4 November 2016; published 7 December 2016)

\begin{abstract}
We study the effect of strong magnetic fields, of the order of $10^{15}-10^{17} \mathrm{G}$, on the extension of the crust of magnetized neutron stars. The dynamical instability region of neutron-proton-electron (npe) matter at subsaturation densities and the mode with the largest growth rate are determined within a relativistic mean-field model. It is shown that the effect of a strong magnetic field on the instability region is very sensitive to the density dependence of the symmetry energy, and that it is at the origin of an increase of the extension of the crust and of the charge content of clusters.
\end{abstract}

DOI: 10.1103/PhysRevC.94.062801

Soft- $\gamma$-ray repeaters and some anomalous $\mathrm{x}$-ray pulsars are strongly magnetized neutron stars known as magnetars [1-3]. These stars have strong surface magnetic fields of the order of $10^{14}-10^{15} \mathrm{G}[4]$, and slow rotation with a period of $\sim 1-12 \mathrm{~s}$. Recently, the time evolution of the magnetic field of isolated x-ray pulsars has been studied by Pons et al. [5]. The authors show that a fast decay of the magnetic field could explain the nonobservation of stars with periods above $12 \mathrm{~s}$. The decay of the magnetic field was obtained by including a high electrical resistivity in the inner crust, attributed to the possible existence of an amorphous and heterogeneous layer at the bottom of the inner crust. The lack of isolated $x$-ray pulsars with a period higher than $12 \mathrm{~s}$ could, therefore, be a direct indication of the existence of an amorphous inner crust, possibly in the form of pasta phases [5].

At low nuclear matter densities, a competition between the long-range Coulomb repulsion and short-range nuclear attraction will lead to the formation of clusterized matter, known as nuclear pasta [6], near the crust-core transition. These geometrical configurations are observed not only in nuclear matter, but also in a variety of amorphous solids, crystals, and magnetic and biological materials [7]. One of the main interests of the existence of these exotic structures in the crust of neutron stars is the effect that they might have on the neutrino transport and the subsequent cooling of the neutron star [8].

Molecular dynamics simulations of the nuclear pasta have shown that topological defects in the pasta could increase electron scattering and reduce the electrical and the thermal conductivities [9]. Electron conductivity in magnetized neutron star matter was also studied in Ref. [10], and it was shown that the electron transport is strongly anisotropic, due to the presence of strong magnetic fields. The complexity introduced by the magnetic field suggests that both suppression and enhancement of the electron conduction in the presence of the pasta phases are possible, and further calculations are required.

\footnotetext{
*jian-junfang@163.com

†pais.lena@gmail.com

${ }^{\ddagger}$ sidney.avancini@ufsc.br

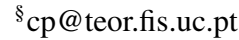

Stellar matter contains, besides neutrons and protons, also electrons, which neutralize the proton charge. The transition clusterized-homogeneous matter has been estimated by using different methods. In particular, Ref. [11] shows that a Thomas-Fermi (TF) description of the pasta phase predicts the same crust-core transition density as a dynamical spinodal calculation, which allows independent electron and proton density fluctuations. The same conclusion was drawn in Ref. [12], where it was shown that the dynamical spinodal calculation gives a lower limit on the crust-core density, and that the larger the isospin asymmetry, the closer this value is to the TF result. For $\beta$-equilibrium matter, both results are practically coincident.

The importance of the thermodynamical and dynamical spinodals on the determination of the behavior of a system that enters an instability region was also pointed out to be connected with nuclear multifragmentation; in particular the time evolution of a compound nucleus during a heavy-ion collision. These instabilities are associated with the density region where the curvature of the free energy is negative $[13,14]$.

Very strong magnetic fields will influence the proton charge fluctuations and, correspondingly, the transport properties [10]. This effect was not considered in the above studies. We will study in the present Rapid Communication the effect of a strong magnetic field on collective modes of stellar matter and dynamical instabilities. This is the first time that such a study is being considered, as far as we know. In a previous work, some of us studied the effect of strong magnetic fields in the nuclear-pasta phase by using relativistic mean field (RMF) models within a TF calculation. However, only magnetic fields above $10^{17} \mathrm{G}$ and large proton fractions were considered [15] and the anomalous magnetic moments (AMM) of protons and neutrons were neglected. Moreover, some results showed abrupt behavior, which was not totally understood.

Here, we restrict ourselves to the longitudinal modes arising from small oscillations around a stationary state in asymmetric nuclear matter at subsaturation densities. This investigation will be performed in the framework of a relativistic meanfield hadronic model within the Vlasov formalism [16-18]. We study the effect of the magnetic field on the spinodal section, the clusterized-homogeneous matter transition, and the average size of clusters in the nonhomogeneous phase. 
Stellar matter is described within the nuclear relativistic mean-field formalism under the effect of strong magnetic fields $[19,20]$, including the effect of the AMM. Nucleons with mass $M$ interact with and through an isoscalar-scalar field $\phi$ with mass $m_{s}$, an isoscalar-vector field $V^{\mu}$ with mass $m_{v}$, and an isovector-vector field $\mathbf{b}^{\mu}$ with mass $m_{\rho}$. Besides nucleons, electrons will also be included in the Lagrangian density. Protons and electrons interact through the electromagnetic field $A^{\mu}$, which includes the static component $A_{\text {stat }}^{\mu}=(0,0, B x, 0)$, so that $\mathbf{B}=B \hat{\mathbf{z}}$ and $\nabla \cdot \mathbf{A}=0$. The static electromagnetic field is assumed to be externally generated, and only frozen-field configurations are considered for this component.

The Lagrangian density (taking $c=\hbar=1$ ) can be written as $\mathcal{L}=\sum_{i=p, n} \mathcal{L}_{i}+\mathcal{L}_{e}+\mathcal{L}_{\sigma}+\mathcal{L}_{\omega}+\mathcal{L}_{\rho}+\mathcal{L}_{\omega \rho}+\mathcal{L}_{A}$, with

$$
\begin{aligned}
& \mathcal{L}_{i}=\bar{\psi}_{i}\left[\gamma_{\mu} i D^{\mu}-M^{*}-\frac{1}{2} \mu_{N} \kappa_{i} \sigma_{\mu \nu} F^{\mu \nu}\right] \psi_{i}, \\
& \mathcal{L}_{e}=\bar{\psi}_{e}\left[\gamma_{\mu}\left(i \partial^{\mu}+e A^{\mu}\right)-m_{e}\right] \psi_{e},
\end{aligned}
$$

where $\quad i D^{\mu}=i \partial^{\mu}-g_{v} V^{\mu}-\frac{g_{\rho}}{2} \boldsymbol{\tau} \cdot \mathbf{b}^{\mu}-e A^{\mu} \frac{1+\tau_{3}}{2}, \quad M^{*}=$ $M-g_{s} \phi, e=\sqrt{4 \pi / 137}$ is the electromagnetic coupling constant, and $\tau_{3}= \pm 1$ is the isospin projection for protons and neutrons, respectively. The nucleon AMM is introduced via the coupling of the baryons to the electromagnetic field tensor with $\sigma_{\mu \nu}=\frac{i}{2}\left[\gamma_{\mu}, \gamma_{\nu}\right]$ and strength $\kappa_{i}$, with $\kappa_{n}=-1.91315$ for the neutron, and $\kappa_{p}=1.79285$ for the proton, and $\mu_{N}$ is the nuclear magneton. As discussed in Ref. [21], the contribution of the AMM of electrons is negligible and is not be considered. For the nuclear matter parameters, we consider the NL3 [22] and NL3 $\omega \rho$ [23] parametrizations, with the symmetry energy slope $L=118$ and $55 \mathrm{MeV}$, respectively. Besides fulfilling

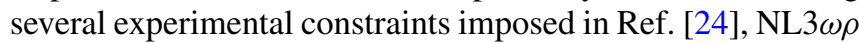
satisfies within a $10 \%$ deviation the constraints imposed by microscopic neutron matter calculations [25] and describes a $2 M_{\odot} \operatorname{star}[26]$.

We determine the dynamical spinodal within the Vlasov formalism discussed in Refs. [16,17]. We denote by $f(\mathbf{r}, \mathbf{p}, t)=\operatorname{diag}\left(f_{p}, f_{n}, f_{e}\right)$ the distribution function for npe matter at position $\mathbf{r}$, instant $t$ and momentum $\mathbf{p}$, and by $h=\operatorname{diag}\left(h_{p}, h_{n}, h_{e}\right)$ the corresponding one-body Hamiltonian, where $h_{i}=\left[\left(\overline{\boldsymbol{p}}_{z}^{i}\right)^{2}+\bar{m}_{i}^{2}\right]^{1 / 2}+\mathcal{V}_{0}^{i}, i=n, p, e$, with $\overline{\boldsymbol{p}}^{i}=\boldsymbol{p}-\mathcal{V}^{i}, \quad \bar{m}_{p}=\left(M_{p}^{* 2}+2 v e B\right)^{1 / 2}-s \mu_{N} \kappa_{p} B, \quad \bar{m}_{n}=$ $\left[M_{n}^{* 2}+\left(\overline{\boldsymbol{p}}_{\perp}^{n}\right)^{2}\right]^{1 / 2}-s \mu_{N} \kappa_{n} B, \bar{m}_{e}=\left(m_{e}^{* 2}+2 v e B\right)^{1 / 2}, \mathcal{V}_{\mu}^{n}=$ $g_{v} V_{\mu}-\frac{g_{\rho}}{2} b_{\mu}, \mathcal{V}_{\mu}^{p}=g_{v} V_{\mu}+\frac{g_{\rho}}{2} b_{\mu}+e A_{\mu}, \mathcal{V}_{\mu}^{e}=-e A_{\mu}$, and $v=n+\frac{1}{2}-\operatorname{sgn}(q) \frac{s}{2}=0,1,2, \ldots$ enumerates the Landau levels of the fermions with electric charge $q$, the quantum number $s$ is $+1(-1)$ for spin parallel (antiparallel) to the magnetic-field direction, taken in the $z$ direction. We define the vectors $(\boldsymbol{p}, \boldsymbol{V}, \ldots)$ along directions parallel $\left(\boldsymbol{p}_{z}, \boldsymbol{V}_{z}, \ldots\right)$ and perpendicular $\left(\boldsymbol{p}_{\perp}, \boldsymbol{V}_{\perp}, \ldots\right)$ to the magnetic field. The time evolution of the distribution function is described by the Vlasov equation

$$
\frac{\partial f_{i}}{\partial t}+\left\{f_{i}, h_{i}\right\}=0, \quad i=p, n, e,
$$

where $\{$,$\} denote the Poisson brackets. From the Euler-$ Lagrange formalism we derive the equations describing the time evolution of the fields $\phi, V^{\mu}, A^{\mu}$ and the third component of the $\rho$ field $b_{3}^{\mu}=\left(b_{0}, \mathbf{b}\right)$.

At zero temperature, the state which minimizes the energy of asymmetric nuclear matter is characterized by the Fermi momenta $P_{F}^{i}, i=p, n, e$ and is described by the distribution function $f_{0}(\mathbf{r}, \mathbf{p})=\operatorname{diag}\left[\Theta\left(P_{F}^{p 2}-p^{2}\right), \Theta\left(P_{F}^{n 2}-p^{2}\right), \Theta\left(P_{F}^{e 2}-\right.\right.$ $\left.p^{2}\right)$ ], where $P_{F}^{p}, P_{F}^{n}, P_{F}^{e}$, are the Fermi momenta of protons, neutrons, and electrons, and by the constant mesonic field equations.

Collective modes correspond to small oscillations around the equilibrium state. These small deviations are described by the linearized equations of motion and the collective modes are the solutions of those equations. Let the deviations from equilibrium be described by $f=f_{0}+\delta f, \phi=\phi_{0}+\delta \phi, V_{0}=$ $V_{0}^{(0)}+\delta V_{0}, V_{i}=\delta V_{i}, b_{0}=b_{0}^{(0)}+\delta b_{0}, b_{i}=\delta b_{i}, A_{0}=\delta A_{0}$, $A_{i}=A_{i, \text { stat }}+\delta A_{i}$. The linearized Vlasov equations for $\delta f_{i}$ are equivalent to the following equations [16]:

$$
\frac{\partial S_{i}}{\partial t}+\left\{S_{i}, h_{0 i}\right\}=\delta h_{i}, \quad i=p, n, e,
$$

where $S_{i}$ are the components of a generating function $S(\mathbf{r}, \mathbf{p})=$ $\operatorname{diag}\left(S_{p}, S_{n}, S_{e}\right)$, such that $\delta f_{i}=\left\{S_{i}, f_{0 i}\right\}$.

In the present work, we consider the longitudinal modes, with momentum $\boldsymbol{k}$ in the direction of the magnetic field and frequency $\omega$, described by the ansatz

$$
\left(\begin{array}{c}
S_{j}(\mathbf{r}, \mathbf{p}, t) \\
\delta \phi \\
\delta \mathcal{B}^{\mu}
\end{array}\right)=\left(\begin{array}{c}
\mathcal{S}_{\omega}^{j}(p, \cos \theta) \\
\delta \phi_{\omega} \\
\delta \mathcal{B}_{\omega}^{\mu}
\end{array}\right) e^{i\left(\omega t-\mathbf{k}_{z} \cdot \mathbf{r}\right)},
$$

where $j=p, n, e, \mathcal{B}=V, b, A$ represents the vector fields, and $\theta$ is the angle between $\boldsymbol{p}$ and $\boldsymbol{k}_{z}$. For these modes, we have $\delta V_{\omega}^{z}=\delta V_{\omega}, \delta b_{\omega}^{z}=\delta b_{\omega}$, and $\delta A_{\omega}^{z}=\delta A_{\omega}$. A set of five independent equations of motion are obtained, in terms of the amplitudes $A_{\omega, j}^{i}$ for the proton and neutron scalar density fluctuations, and the proton, neutron and electron vector density fluctuations. The eigenmodes $\omega$ of the system are the solutions of the dispersion relation obtained, equating to zero the determinant of the matrix of the coefficients of the five equations of motion.

The region in $\left(\rho_{p}, \rho_{n}\right)$ space for a given wave vector $\boldsymbol{k}$, satisfying $\omega=0$, defines the dynamical spinodal surface. At low densities, the system presents unstable modes, characterized by an imaginary frequency. Inside the unstable region, the mode with the largest growth rate $\Gamma$, such that $\omega=i \Gamma$, is the one that drives the system to the formation of instabilities. Its half wavelength gives a good estimate of the most probable size of the clusters (liquid) formed in the mixed (liquid-gas) phase [18]. In the following, possible effects of strong magnetic fields on the structure of the inner crust of magnetars are discussed from the analysis of the dynamical spinodal surface and the unstable modes with the largest growth rate. This approach takes into account finite-size effects, such as the surface tension and Coulomb effects.

The most intense fields detected on the surface of a magnetar are not larger than $2 \times 10^{15} \mathrm{G}$, the smallest field we consider. We may, however, expect stronger fields in the interior. In particular, toroidal fields more intense than $10^{17} \mathrm{G}$ have been obtained in stable configurations [27,28]. 


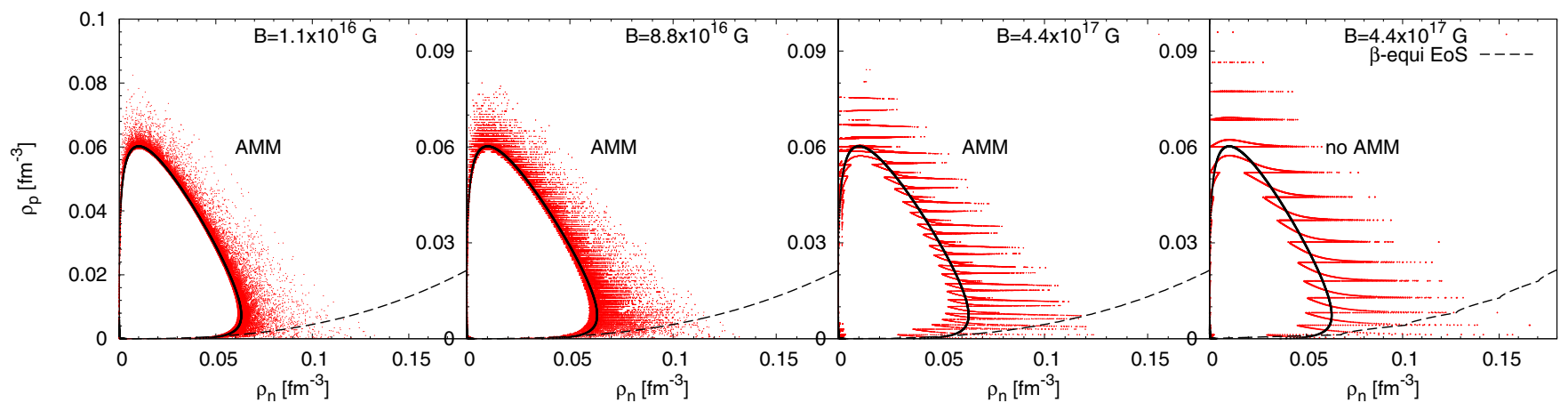

FIG. 1. Dynamical spinodals for the NL3 parametrization, for different magnetic-field intensities and momentum transfer $k=50 \mathrm{MeV}$.

In Fig. 1, we show the spinodal sections in the $\left(\rho_{p}, \rho_{n}\right)$ space obtained with the NL3 parametrization for the magnetic fields $1.1 \times 10^{16} \mathrm{G}, 8.8 \times 10^{16} \mathrm{G}$, and $4.4 \times 10^{17} \mathrm{G}$, calculated for a wave number $k=50 \mathrm{MeV}$, which gives a spinodal section close to the envelope of all spinodal sections. The calculations were carried out including AMM, except for the largest field, for which we also show the no AMM spinodal. Due to a numerical limitation, the spinodal sections are made of points, which, however, define close regions. Each point is a solution of dispersion relation obtained for a fixed proton fraction which varies between 0 and 1 . The thick black line represents the spinodal section for a zero magnetic field. The dashed line is the EoS of $\beta$-equilibrium matter and allows the identification of the crust-core transition.

In the right panel of Fig. 1, we can see that a magnetic field equal to $4.4 \times 10^{17} \mathrm{G}$ is strong enough to create bands of instability at densities above $0.05 \mathrm{fm}^{-3}$, associated with the filling of the different Landau levels. In Ref. [29], the thermodynamical spinodal section, which corresponds to the $k=0$ limit of the dynamical spinodal, excluding electrons and the Coulomb field, was studied for magnetic fields equal or above $5 \times 10^{18} \mathrm{G}$. Spinodal bands are also present, although the stronger the field, the smaller the number of bands. The appearance of bands was attributed to the behavior of the proton chemical potential with density within each Landau band: at the bottom of the band it has a very soft behavior; however, at the top of the Landau level it hardens and a cusp occurs when a new Landau level opens, followed by a softening of the chemical potential. The proton and neutron AMM give rise to extra bands.

There are mainly two contributions for the spinodal section: (a) a closed region that contains the $B=0$ spinodal and extra regions that form spike-like structures, associated with the filling of Landau levels, and (b) disconnected regions that appear with the opening of new Landau levels at densities well above the $B=0$ crust-core transition density. The maximum growth rates at constant proton fraction (see Fig. 2) allow a more clear picture: there is a closed region that, although with some fluctuations, follows the $B=0$ curve, followed by separate regions, whose density width decreases continuously until homogeneous matter sets in. These disconnected regions appear when a new Landau level starts being filled.

In Ref. [29], it was shown that the extension of the thermodynamical spinodal for $\rho_{p}=0$ was independent of $B$, with the border to homogeneous matter at $\rho_{n}^{N L 3}=0.213 \mathrm{fm}^{-3}$ and $\rho_{n}^{N L 3 \omega \rho}=0.122 \mathrm{fm}^{-3}$ for the models we consider in the present study. These numbers set an upper limit of the extension of the dynamical spinodal, which in fact is too high, because matter in the stars has a finite proton fraction: for NL3 $(\mathrm{NL} 3 \omega \rho)$ and the proton fraction at the crustcore transition, $y_{p}^{N L 3}=0.02\left(y_{p}^{N L 3 \omega \rho}=0.035\right)$, the dynamical spinodal extension is reduced to $\rho \sim 0.16(0.115) \mathrm{fm}^{-3}$ for $B=4.4 \times 10^{16} \mathrm{G}$. Decreasing further the magnetic field to $2.2 \times 10^{14} \mathrm{G}$, the extension of the spinodal decreases to $0.105 \mathrm{fm}^{-3}$ for NL3, and $0.102 \mathrm{fm}^{-3}$ for NL3 $\omega \rho$, showing a convergence to the $B=0$ result, respectively 0.056 and $0.084 \mathrm{fm}^{-3}$.

It was shown in Ref. [11] that the size of the clusters in the inner crust of a neutron star, calculated within the TF framework for RMF models, is well estimated by the half wavelength associated with the most unstable mode, i.e., the one that drives matter into a nonhomogeneous phase [30]. In the same work, it was also shown that for NL3 the average proton fraction in the inner crust, for densities above $0.01 \mathrm{fm}^{-3}$,
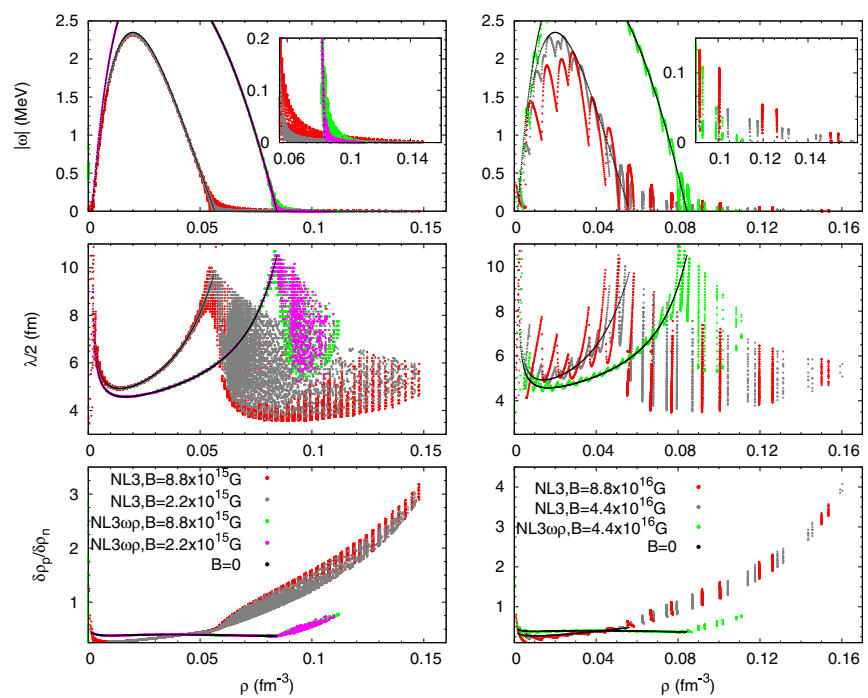

FIG. 2. Largest growth rate $\Gamma=|\omega|$ (top panels), the corresponding half-wavelength (middle panels), and the proton-neutron density fluctuation ratio (bottom panels) versus density, for different magnetic-field intensities and matter with $y_{p}=0.02$ for NL3 and $y_{p}=0.035$ for NL3 $\omega \rho$. The black curve corresponds to the $B=0$ results. 
is $y_{p} \sim 0.02$, while for NL3 $\omega \rho$ is $y_{p}=0.035$. We have, therefore, determined the most unstable modes for magnetized matter for both parametrizations with the corresponding proton fractions, in order to get an estimation of the size and change of charge content of the clusters formed in the inner crust.

In Fig. 2, the largest growth rates (top panels), the corresponding half wavelength (middle panels) and the ratio $\delta \rho_{p} / \delta \rho_{n}$ between the proton and neutron density fluctuations (bottom panels) are shown for fields between $B=2.2 \times 10^{15}$ $\mathrm{G}$ and $8.8 \times 10^{16} \mathrm{G}$ for NL3 and NL3 $\omega \rho$. In all figures, the $B=0$ results are represented by a black curve. First, we consider the strongest field, represented by red dots in the right panel of Fig. 2, obtained only for NL3. In this case, there are several well-defined regions of clusterized matter separated by regions of homogeneous matter. This is a consequence of the bands of instability, due to the filling of the Landau levels. Also, the size of the clusters is affected. In the first region of instability, the size of the clusters oscillates around the results for $B=0$, and fast size changes occur in a very small density interval. After the first instability region, several others appear, although the larger the density, the smaller the density width of each region. Considering weaker fields, all these features are repeated with a denser appearance of unstable regions but with smaller widths each. The transition density to homogeneous matter is changing slowly with the magnetic-field intensity, but considering sufficiently small fields, the finite- $B$ spinodal converges to the $B=0$ one, as discussed before. This convergence is reflected on the decrease of the magnitude of the growth rate at a density $10 \%$ larger than the $B=0$ crust-core transition density with a decrease of the magnetic field: for NL3 (NL3wr), it goes from 0.3855 $(0.1481) \mathrm{MeV}$ at $4.4 \times 10^{16} \mathrm{G}$, to $0.0921(0.0378) \mathrm{MeV}$ for $8.8 \times 10^{15} \mathrm{G}$ and $0.0068(0.0020) \mathrm{MeV}$ for $4.4 \times 10^{14} \mathrm{G}$.

The extension of the region with disconnected unstable regions is strongly dependent on the density dependence of the symmetry energy: for the NL3 $\omega \rho$ parametrization with $L=55 \mathrm{MeV}$, the unstable region extends only until $\rho=0.113 \mathrm{fm}^{-3}$. This increases to $\rho \sim 0.12,0.13,0.16 \mathrm{fm}^{-3}$, respectively, for $L=68,88,118 \mathrm{MeV}$ and stellar matter conditions. Taking a larger proton fraction, $y_{p}=0.1$ which may be more realistic at larger densities, there will still appear unstable regions for $\rho \leqslant 0.11 \mathrm{fm}^{-3}$ for NL3 $\omega \rho$ with $L=$ $55 \mathrm{MeV}$ and $\rho \leqslant 0.135 \mathrm{fm}^{-3}$ for NL3, with $L=118 \mathrm{MeV}$.

The proton-neutron density fluctuation ratio was also calculated. Although $y_{p}=0.02$ corresponds to $\rho_{p} / \rho_{n}=1 / 49$, the fluctuations give rise to clusterized matter with a much larger proton content: above the $B=0$ crust-core transition density the fluctuations $\delta \rho_{p} / \delta \rho_{n}$ increase from $\sim 0.35$ to more than the double for NL3 $\omega \rho$, and a factor of 5 for NL3, see both panels of Fig. 2.

In conclusion, we have shown that Landau levels originate a spinodal section with a structure of bands, with disconnected regions for the larger densities. We expect that this irregular border, including disconnected regions, will give rise to a more heterogeneous and amorphous phase of matter than the one already expected due to the formation of the pasta phases. Studies of electron conduction in this matter are needed to confirm whether it would reduce electron conductivity, and thus originate a resistive layer within the crust of magnetized neutron stars, as proposed in Ref. [5].

The study was complemented with the determination of the largest growth rate inside the spinodal surface, which has allowed us to estimate the size of the clusters formed, when the system is driven into a nonhomogeneous phase. It was shown that, close to the transition to homogeneous matter, there is a heterogeneous region, alternating nonhomogeneous and homogeneous matter, with proton-richer clusters. Inside the spinodal section, the average size of the clusters and its proton content vary in an oscillatory way, reinforcing the heterogeneity of the inner crust matter. All these effects are very sensitive to the density dependence of the symmetry energy.

To conclude, we refer another pulsar property that could be affected by an increase of the crust. Pulsar glitches are attributed to the angular-momentum transfer between the crust and the core [31], involving the vortex dynamics associated with the neutron superfluid confined to the inner crust. However, the recent detection of an antiglitch [32], or the indication that due to entrainment the inner crust angular moment is not enough to explain the glitch mechanism [33], suggests that the glitch theory has to be clarified. The effects of the magnetic field on the inner crust, in particular, an increase of the crust, the succession of clusterized and homogeneous layers, and a nonmonotonic change of the neutron gas background density will certainly affect the glitch mechanism and should be taken into account in a glitch theory.

We thank Isaac Vidaña for useful discussions. This work is partly supported by the FCT (Portugal) projects PEstOE/FIS/UI0405/2014, developed under the initiative QREN, and UID/FIS/04564/2016, by COST Action MP1304 "NewCompStar", and by CNPq. H.P. is supported by FCT under Project No. SFRH/BPD/95566/2013.
[1] R. C. Duncan and C. Thompson, Astrophys. J. 392, L9 (1992); C. Thompson and R. C. Duncan, Mon. Not. R. Astron. Soc. 275, 255 (1995).

[2] V. V. Usov, Nature (London) 357, 472 (1992).

[3] B. Paczynski, Acta Astron. 42, 145 (1992).

[4] SGR/APX online catalogue; http://www.physics.mcgill.ca/ $\sim$ pulsar/magnetar/main.html.

[5] J. A. Pons, D. Viganò, and N. Rea, Nat. Phys. 9, 431 (2013).

[6] D. G. Ravenhall, C. J. Pethick, and J. R. Wilson, Phys. Rev. Lett. 50, 2066 (1983); C. J. Horowitz, M. A. Pérez-García, and
J. Piekarewicz, Phys. Rev. C 69, 045804 (2004); H. Sonoda, G. Watanabe, K. Sato, K. Yasuoka, and T. Ebisuzaki, ibid. 77, 035806 (2008); A. S. Schneider, D. K. Berry, C. M. Briggs, M. E. Caplan, and C. J. Horowitz, ibid. 90, 055805 (2014).

[7] G. Watanabe and H. Sonoda, in Soft Condensed Matter: New Research, edited by K. I. Dillon (Nova Science Publishers, New York, 2007), arXiv:cond-mat/0502515; R. Moessner and A. P. Ramirez, Phys. Today 59, 24 (2006); O. Kuksenok, R. D. M. Travasso, and A. C. Balazs, Phys. Rev. E 74, 011502 (2006); 
Y. Han, Y. Shokef, A. M. Alsayed, P. Yunker, T. C. Lubensky, and A. G. Yodh, Nature (London) 456, 898 (2008); T. Bánsági Jr., V. K. Vanag, and I. R. Epstein, Science 331, 1309 (2011).

[8] C. J. Horowitz, M. A. Pérez-García, J. Carriere, D. K. Berry, and J. Piekarewicz, Phys. Rev. C 70, 065806 (2004); H. Sonoda, G. Watanabe, K. Sato, T. Takiwaki, K. Yasuoka, and T. Ebisuzaki, ibid. 75, 042801(R) (2007); M. D. Alloy and D. P. Menezes, ibid. 83, 035803 (2011).

[9] C. J. Horowitz, D. K. Berry, C. M. Briggs, M. E. Caplan, A. Cumming, and A. S. Schneider, Phys. Rev. Lett. 114, 031102 (2015).

[10] D. G. Yakovlev, Mon. Not. R. Astron. Soc. 453, 581 (2015).

[11] S. S. Avancini, D. P. Menezes, M. D. Alloy, J. R. Marinelli, M. M. W. Moraes, and C. Providência, Phys. Rev. C 78, 015802 (2008).

[12] S. S. Avancini, S. Chiacchiera, D. P. Menezes, and C. Providência, Phys. Rev. C 82, 055807 (2010); 85, 059904(E) (2012).

[13] H. Müller and B. D. Serot, Phys. Rev. C 52, 2072 (1995).

[14] P. Chomaz, M. Colonna, and J. Randrup, Phys. Rep. 389, 263 (2004).

[15] R. C. R. de Lima, S. S. Avancini, and C. Providência, Phys. Rev. C 88, 035804 (2013).

[16] M. Nielsen, C. Providência, and J. da Providência, Phys. Rev. C 44, 209 (1991).

[17] C. Providência, L. Brito, S. S. Avancini, D. P. Menezes, and Ph. Chomaz, Phys. Rev. C 73, 025805 (2006).

[18] L. Brito, C. Providência, A. M. Santos, S. S. Avancini, D. P. Menezes, and Ph. Chomaz, Phys. Rev. C 74, 045801 (2006).
[19] A. Broderick, M. Prakash, and J. M. Lattimer, Astrophys. J. 537, 351 (2000).

[20] A. Rabhi, C. Providência, and J. da Providência, J. Phys. G 35, 125201 (2008).

[21] Robert C. Duncan, AIP Conf. Proc. 526, 830 (2000).

[22] G. A. Lalazissis, J. König, and P. Ring, Phys. Rev. C 55, 540 (1997).

[23] C. J. Horowitz and J. Piekarewicz, Phys. Rev. Lett. 86, 5647 (2001); Phys. Rev. C 64, 062802 (2001).

[24] M. Dutra, O. Lourenço, S. S. Avancini, B. V. Carlson, A. Delfino, D. P. Menezes, C. Providência, S. Typel, and J. R. Stone, Phys. Rev. C 90, 055203 (2014).

[25] S. Gandolfi, J. Carlson, and Sanjay Reddy, Phys. Rev. C 85, 032801(R) (2012); K. Hebeler, J. M. Lattimer, C. J. Pethick, and A. Schwenk, Astrophys. J. 773, 11 (2013).

[26] M. Fortin, C. Providência, A. R. Raduta, F. Gulminelli, J. L. Zdunik, P. Haensel, and M. Bejger, Phys. Rev. C 94, 035804 (2016).

[27] K. Kiuchi and S. Yoshida, Phys. Rev. D 78, 044045 (2008).

[28] J. Frieben and L. Rezzolla, Mon. Not. R. Astron. Soc. 427, 3406 (2012).

[29] A. Rabhi, C. Providência, and J. Da Providência, Phys. Rev. C 79, 015804 (2009).

[30] A. M. Santos, L. Brito, and C. Providência, Phys. Rev. C 77, 045805 (2008).

[31] B. Link, R. I. Epstein, and J. M. Lattimer, Phys. Rev. Lett. 83, 3362 (1999).

[32] R. F. Archibald et al., Nature (London) 497, 591 (2013).

[33] N. Andersson, K. Glampedakis, W. C. G. Ho, and C. M. Espinoza, Phys. Rev. Lett. 109, 241103 (2012). 